\title{
Targeted Therapy for Patients With Metastatic Non-Small Cell Lung Cancer
}

\author{
Presented by Karen L. Reckamp, MD, MS
}

\section{Abstract}

Molecular testing is recommended for initial diagnosis in patients with non-small cell lung cancer (NSCLC), according to the updated NCCN Guidelines, because targeted therapies are available that can improve patient outcomes. Targeted therapies are currently approved for EGFR mutations, ALK and ROS1 gene rearrangements, and BRAF mutations, with the list of emerging "actionable" targets growing. The 2018 NCCN Guidelines for NSCLC incorporate new therapies, including the EGFR tyrosine kinase inhibitor osimertinib and the ALK inhibitor alectinib, as first-line preferences.

J Natl Compr Canc Netw 2018;16(5.5):601-604 doi: 10.6004ljnccn.2018.0046

\begin{abstract}
"There is a growing list of approved targets and therapies included in the 2018 NCCN Guidelines for NonSmall Cell Lung Cancer [NSCLC] as well as emerging targets. Why does this matter? Because targeted therapies improve survival," said Karen L. Reckamp, MD, MS, Co-Director, Lung Cancer and Thoracic Oncology Program, and Professor, City of Hope Comprehensive Cancer Center.

"With no targeted therapy, median survival for a select group of patients with NSCLC is 2 years. If there is an oncologic driver and no targeted therapy is given, median overall survival [OS] is approximately 2 years. But, when there is an oncologic driver and targeted therapy is given, median OS jumps to over 3 years," she told the audience at the NCCN 23rd Annual Conference. ${ }^{1}$

The list of mutations with approved targeted therapies includes EGFR mutations (present in 30\%-40\% of Asians ${ }^{2}$ and $10 \%-20 \%$ of Caucasians), ${ }^{3}$ ALK rearrangements (present in up to $7 \%$ ), ROS1 rearrangements

\footnotetext{
Presented by Karen L. Reckamp, MD, MS, City of Hope Comprehensive Cancer Center, Duarte, California.

Dr. Reckamp has disclosed that she has received grant/research support from AbbVie; ACEA; Adaptimmune; ARIAD Pharmaceuticals, Inc.; Boehringer Ingelheim GmbH; Bristol-Myers Squibb Company; Genentech, Inc.; Guardant; Loxo Oncology; Novartis Pharmaceuticals Corporation; Pfizer Inc.; Seattle Genetics; and Xcovery. She has also received consulting fees/honoraria from Amgen Inc., ARIAD Pharmaceuticals, Inc., Astellas, Euclises, and Tesaro, Inc.

Correspondence: Karen L. Reckamp, MD, MS, City of Hope Comprehensive Cancer Center, 1500 East Duarte Road, Building 51, Duarte, CA 91010. Email: kreckamp@coh.org
}

(1.7\%), and BRAF mutations (present in $2 \%$ ). Other targets with therapies that are not approved for use in NSCLC include HER2 (present in 2\%), RET (present in $1.7 \%$ ), MET (present in 10\%), and NTRK (present in $<1 \%$ ).

The 2018 NCCN Guidelines recommend molecular testing to make the initial diagnosis, and this is then followed by PD-L1 testing. For adenocarcinoma, testing is recommended for EGFR, ALK, ROS1, and BRAF mutations as part of broad molecular profiling, in addition to PD-L1 testing. For squamous cell carcinoma, the recommendation is to consider testing for the same 4 mutations in a subset of patients and to perform PD-L1 testing in all patients.

\section{EGFR Mutations}

It has been established that EGFR tyrosine kinase inhibitors (TKIs) work well for patients with EGFR mutations, improving progression-free survival (PFS) and response rates. Yet, EGFR TKIs have varying toxicities.

The 2018 NCCN Guidelines have added osimertinib to the list of recommended drugs for first-line therapy in patients with EGFR mutations discovered prior to first-line therapy, which includes erlotinib, afatinib, gefitinib (all category 1), and osimertinib (recently FDA-approved for the first line).

Dacomitinib, a second-generation EGFR TKI, is not FDA-approved but has encouraging data. The phase 
III ARCHER 1050 trial compared dacomitinib versus gefitinib in 452 patients with advanced NSCLC and an EGFR-activating mutation. ${ }^{4}$ According to a blinded independent review in an intent-to-treat analysis, median PFS was 14.7 months for dacomitinib versus 9.2 for gefitinib $(P<.0001) .{ }^{4}$ Thus, toxicity is greater with the second-generation EGFR TKIs, including afatinib.

"With so many drugs to use as frontline therapy, our patients want to know which drug is best as firstline therapy. Now, we have more data and can add the third-generation EGFR TKI osimertinib to the mix," Dr. Reckamp continued.

The phase III FLAURA trial compared osimertinib versus erlotinib or gefitinib as first-line therapy. ${ }^{7}$ PFS was significantly improved with osimertinib. Median PFS was 18.9 months with osimertinib versus 10.2 with standard of care $(P<.0001)$ (Figure 1). Osimertinib is effective in patients with mutant EGFR and in those with EGFR wild-type. Further, osimertinib improved PFS in patients with and without brain metastases, and has less toxicity than either the first- or second-generation EGFR TKIs.

Virtually all patients who experience response to frontline EGFR TKI therapy develop resistance. The most common mechanism of acquired resistance is the T790M mutation ( $\approx 50 \%$ of patients). Osimertinib is FDA-approved for treatment of acquired T790M re-

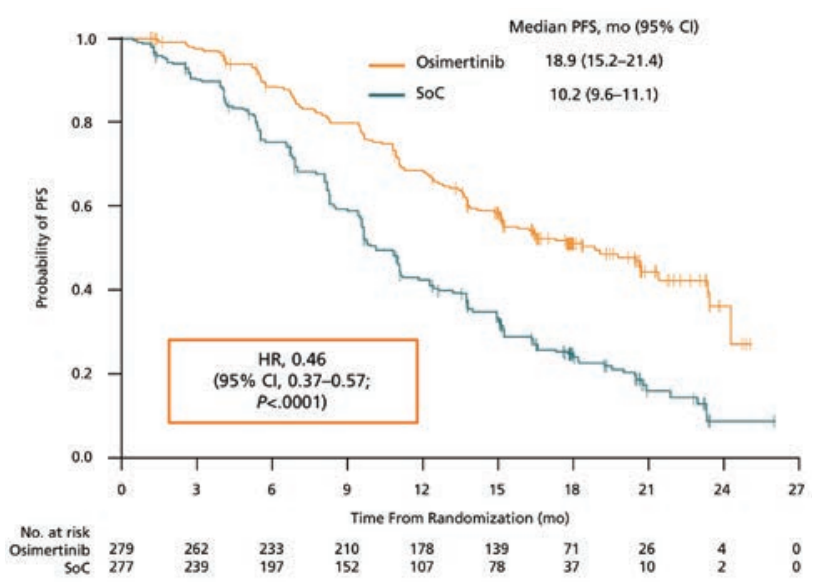

Figure 1. Primary end point: PFS by investigator assessment. There were 342 events in 556 patients at data cut-off; $62 \%$ reached maturity. For osimertinib there were 136 events $(49 \%)$, and for SoC there were 206 events (74\%). Tick marks indicate censored data.

Abbreviations: HR, hazard ratio; PFS, progression-free survival; SoC, standard of care.

From Soria JC, Ohe Y, Vansteenkiste J, et al. Osimertinib in untreated EGFRmutated advanced non-small-cell lung cancer. N Engl J Med 2018;378:113125. Copyright $\odot 2018$ Massachusetts Medical Society. Reprinted with permission. sistance. Other mechanisms of resistance in patients with activating EGFR mutations are less well understood and include HER2 mutations (8\%), HER2 plus T790M (4\%), MET amplification, small cell lung cancer transformation, and unknown (18\%).

"We believe acquired resistance will be different with osimertinib," she stated.

Currently, most US patients with EGFR mutations receive first- or second-generation EGFR TKIs as standard of care and $>50 \%$ develop T790M as a mechanism of acquired resistance. When osimertinib, as an inhibitor of T790M, is used as first-line therapy, patients will not develop T790M as a primary mechanism of resistance and other patterns of resistance are expected.

Lung cancer management is moving toward bloodbased testing (ie, liquid biopsy). Patients had similar responses to osimertinib whether T790M was positive in blood or tissue, Dr. Reckamp explained. Blood tests may provide false-negative results, but a positive blood (plasma) test is equivalent to a tissue-based test.

\section{Sequencing Therapy}

With numerous good treatment options available, there is much discussion about how to sequence therapy. Currently, first- or second-generation EGFR TKIs are used as first-line therapy, with osimertinib following if $\mathrm{T} 790 \mathrm{M}$ is present on testing at resistance. T790M-negative patients move on to chemotherapy in the second line. If osimertinib is used in the first line, chemotherapy is generally given in the second line, and it is not known whether to use firstand second-generation EGFR TKIs in the third line. "With more data, second-line therapy will change," Dr. Reckamp said.

\section{ALK-Rearranged NSCLC}

The biggest change in the 2018 NCCN Guidelines for patients with ALK gene rearrangements is the recommendation for alectinib as preferred first-line therapy over crizotinib or ceritinib (category 1); crizotinib and ceritinib remain category 1 choices. This recommendation is based on the recent ALEX and J-ALEX trials.

ALEX compared alectinib versus crizotinib in 286 patients with ALK-positive NSCLC. ${ }^{6} \mathrm{Me}$ dian PFS was not reached with alectinib versus 11 


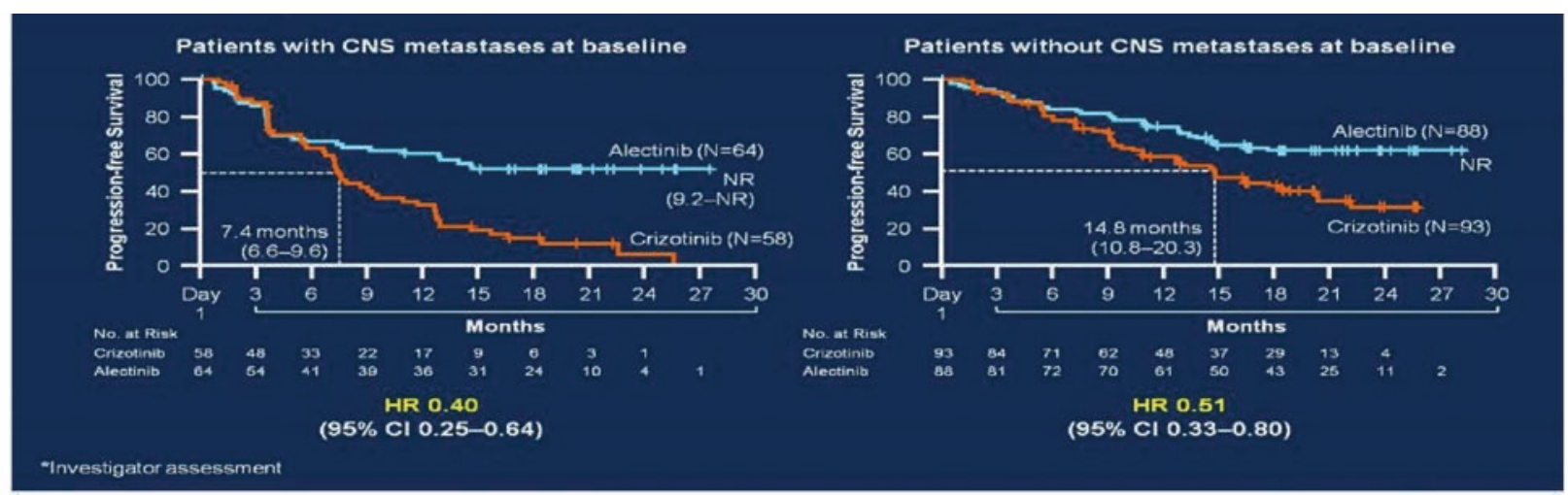

Figure 2. PFS by baseline CNS metastases status.

Abbreviations: CNS, central nervous system; HR, hazard ratio; NR, not reached; PFS, progression-free survival.

From Shaw AT, Peters S, Mok T, et al. Alectinib versus crizotinib in treatment-naive advanced ALK-positive non-small cell lung cancer (NSCLC): primary results of the global phase III ALEX study [abstract]. J Clin Oncol 2017;35(Suppl):Abstract LBA9008; with permission.

months with crizotinib $(P<.0001)$. Alectinib remained significantly superior to crizotinib in terms of PFS in patients with and without brain metastases, ${ }^{6}$ with a median PFS not reached for alectinib compared with 7.4 months for crizotinib in those with brain metastases, versus not reached for alectinib and 14.8 months for crizotinib in those without (Figure 2).

Alectinib's activity in central nervous system (CNS) metastases confirmed data from the J-ALEX trial in Japanese patients. ${ }^{7}$ OS is still immature in both the ALEX and J-ALEX trials.

"This moves alectinib to the first line for ALKpositive NSCLC. The toxicity is manageable, the CNS penetration excellent, and it is neuroprotective in patients without CNS metastasis. There is no question that alectinib should be first line. We await OS results. These data also tell us that patients on crizotinib should be monitored for CNS progression as routine surveillance," Dr. Reckamp stated.

Different mechanisms of resistance have been described with second-and third-generation ALKTKIs. "At this time, testing for resistance mechanisms in patients with ALK gene rearrangements is not standard of care, but this is feasible as we move to liquid biopsy. I think the patterns of resistance will change when alectinib is used as frontline therapy," she noted.

The 2018 NCCN Guidelines include recommendations for progression on crizotinib, alectinib,

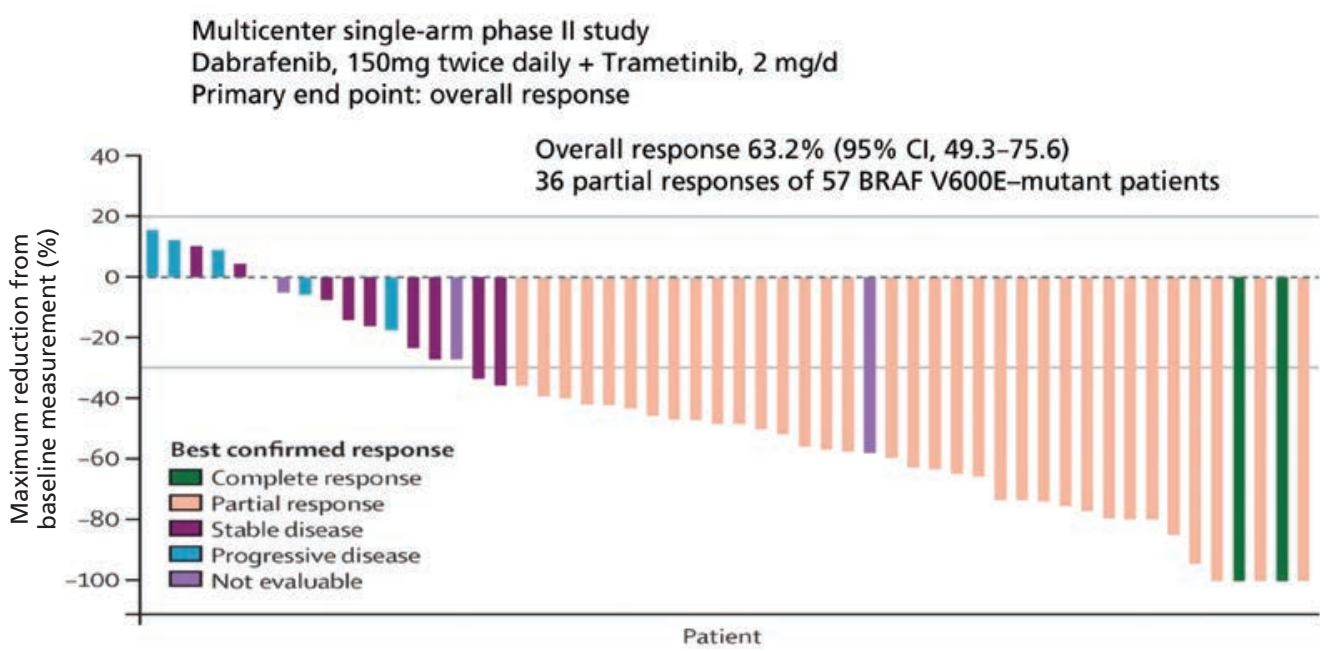

Figure 3. Dabrafenib plus trametinib in $B R A F$ V600E-mutant lung cancer.

Reprinted from Planchard D, Besse B, Goren H, et al. Dabrafenib plus trametinib in patients with previously treated BRAF V600E-mutant metastatic non-small cell lung cancer: an open-label, multicentre phase 2 trial. Lancet Oncol 2016;14:984-993, () 2016, with permission from Elsevier. 
or ceritinib based on whether the patient is asymptomatic or symptomatic.

\section{Other Mutations}

ROS1 rearrangements are found in some patients with NSCLC and do not overlap with ALK translocations. According to the 2018 NCCN Guidelines for NSCLC, crizotinib is the preferred treatment but ceritinib can also be used.

Another addition to the guidelines is the combination of dabrafenib and trametinib for the treatment of BRAF mutations, which occur in $1 \%-3 \%$ of patients with NSCLC. Responses to this combination are in the $60 \%$ range, Dr. Reckamp noted (Figure 3).

"With multiple approved targeted therapies, performing multiplex testing is incredibly important for NSCLC. All patients with nonsquamous disease should be tested, as should those with squamous disease who are nonsmokers or have small tissue samples. There are multiple treatment options, including PD-L1-targeted therapies, but that is another discussion. It is important to test for targets to select the best treatment," she elaborated.

The 2018 NCCN Guidelines include several emerging targets, such as MET-altered lung cancer (high-level MET amplification or MET exon 14 skipping mutation can be treated with crizotinib), RET rearrangements (can be treated with cabozantinib or vandetanib), and HER2 mutations (can be treated with ado-trastuzumab emtansine).

NTRK fusions are not included in the guidelines, but once a drug is approved to target these alterations, this is likely to be included as well because promising data was recently presented. ${ }^{8}$

\section{References}

1. Kris MG, Johnson BE, Berry LD, et al. Using multiplexed assays of oncogenic drivers in lung cancers to select targeted drugs JAMA 2014;11:1998-2006.

2. Mok TS, Wu YL, Thongprasert S, et al. Gefitinib or carboplatin-paclitaxel in pulmonary adenocarcinoma. N Engl J Med 2009;361:947-957.

3. Rosell R, Moran T, Queralt C, et al. Screening for epidermal growth factor receptor mutations in lung cancer. N Engl J Med 2009;361:958-967.

4. Wu YL, Cheng Y, Zhou X, et al. Dacomitinib versus gefitinib as first-line treatment for patients with EGFR-mutation-positive non-small-cell lung cancer (ARCHER 1050): a randomized, open-label, phase 3 trial. Lancet Oncol 2017;11:1454-1466.

5. Soria JC, Ohe Y, Vansteenkiste J, et al. Osimertinib in untreated EGFR-mutated advanced non-small-cell lung cancer. N Engl J Med 2018;378:113-125.

6. Peters S, Camidge DR, Shaw AT, et al. Alectinib versus crizotinib in untreated ALK-positive non-small-cell lung cancer. N Engl J Med 2017;377:829-838.

7. Hida $\mathrm{T}$, Nokihara $\mathrm{H}$, Kondo $\mathrm{M}$, et al. Alectinib versus crizotinib in patients with ALK-positive non-small-cell lung cancer (J-ALEX): an open-label, randomised phase 3 trial. Lancet 2017;390:29-39.

8. Drilon A, Laetsch TW, Kummar S, et al. Efficacy of lacotrectinib in TRK fusion-positive cancers in adults and children. $\mathrm{N}$ Engl J Med 2018;378:731-739. 